\title{
Creating, Growing and Sustaining Efficient Innovation Teams
}

\section{Casimer DeCusatis}

Economic forces such as the growing service economy and commoditization of traditional value chains have led many organizations to pursue breakthrough innovations as part of their business strategy. There has been an increased interest in collaboration and teamwork as catalysts of innovation, often without a clear understanding of the different kinds of teams that can be used to foster innovation or the kinds of team building that will be most likely to yield desired results. The author describes a framework for innovation teams, ranging from highly structured to spontaneous, giving examples of how different kinds of teams relate to the characteristics of the next generation of innovators. A case study illustrates how one approach using preference profiling is more likely to yield tangible results from an innovation team.

\section{Introduction: The Changing Nature of Innovation}

$\mathrm{T}$ The global innovation - commoditization duality has never been more pronounced than in our current economy. Many organizations are investing in efforts designed to promote innovation, without a clear idea of how these investments translate into business value. Furthermore, organizations are also evolving from an industrial base to a service base. This is driven by several factors, including the removal of barriers to service relationships brought about by the virtualization and dissemination of information. However, value capture in the service market is based on customer perception and utility rather than more traditional metrics such as cost and quality (Ho, 2008). Consequently, the value created through innovative service teams also goes largely unrecognized, making it difficult to assess the impact of teams charged with producing innovative results.

There is a great body of literature on the theory of innovation, how people collaborate and the role, structure and types of innovation ecosystems that occur (Rhodes, 1961; Johnson, 1972; Isaksen, Dorval \& Treffinger, 1994; Davila, Epstein \& Shelton, 2006). While many companies consider themselves innovative, most lack a common lexicon for understanding how their investments in innovation trans- late into business value. In particular, while it is recognized that collaboration is an important element of innovation, there is a need for better approaches to forming, growing and sustaining teams of innovators. After reviewing the changing nature of innovation and the emerging generation of innovators, we propose a framework for classifying innovation teams. (Note: the pronoun 'we' is used throughout the paper to refer to the task force that carried out the internal IBM innovation study that this paper is based on.) This allows us to better match the characteristics of the team with the approach to innovation, making it more likely to achieve the desired results.

It is important to first recognize that the fundamental nature of innovation has been changing in recent years. There is a growing emphasis on collaboration as part of the innovation process. There are sound economic reasons why collaborations are growing in importance, including the rising cost of technology development, shortening product lifecycles, and the difficulty in sustaining closed research and development (R\&D) models. An increased focus on core competencies at many businesses has provided an opportunity for interdependencies to a much greater degree than at any time previously. As global information networks make knowledge increasingly widespread, social networking tools 


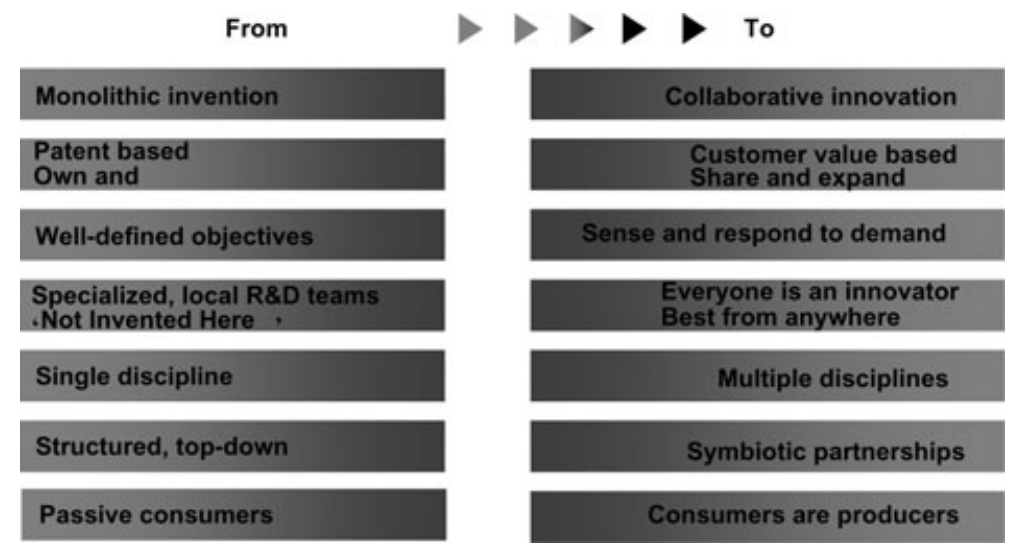

Figure 1. The Changing Nature of Innovation

(Web 2.0 and 3.0) create more opportunities for like-minded parties to find each other and for interdisciplinary teams to form in unexpected ways. In many technology-based industries, the traditional value chain is breaking down; faced with diminishing returns on their $R \& D$ or venture capital investments, many companies have begun to emphasize collaborative tools as a catalyst for innovation. We conceptualize this view using the model illustrated in Figure 1, which distinguishes between two types of innovation approaches that we call monolithic and collaborative.

The monolithic approach represents the conventional view of innovation, as driven by large organizations that hold an effective monopoly on their markets. Innovations are created by a relatively small group of discipline-specific experts, working under controlled conditions with specialized equipment. The problems they address are typically fairly well defined, and their solutions represent highly valued intellectual capital which is protected by patents. Innovations proceed through the development process in a linear way, eventually reaching a group of passive consumers. Feedback is limited to a sampling of customer opinion in between product development cycles. This approach has held sway in the technology industry for many decades; funding for corporate research and development labs is based on the business value produced by this approach. While we can demonstrate that this approach still works well under some conditions (specifically when there is an effective monopoly), a new approach has emerged within the past decade or so which we call collaborative innovation. In its purest form, this differs significantly from the monolithic model. Collaborative innovation delivers customer value through the creation of relationships and social networks, which involve customers early in the development process and maintain their involvement continuously. Valuable ideas can come from anywhere, at any time, and be incorporated into the product based solely on their merit. Such collaboration is interdisciplinary and cuts across organization silos. Intellectual capital is shared freely; indeed, since we may be unable to determine exactly when an idea was first conceived or by whom, the concept of patents breaks down. Some Internet-based companies, universities, and a few others have fully embraced this model. Most organizations, however, fall somewhere in between these two extremes, sharing characteristics of both approaches or changing their focus for different projects.

An example from the computer industry helps to illustrate the migration from monolithic to collaborative approaches as an innovation driver in business. Within IBM, consider the mainframe tradition spanning Systems $360,370,390$, and Z; this began as a monoculture many years ago, and became quite successful, coming to dominate the Fortune 500 market (particularly the financial sector). Starting in the early 1960s, innovation on the mainframe was driven exclusively through corporate $R \& D$, and consisted mainly of delivering anticipated, incremental improvements to the processor speed, memory and other performance benchmarks on a regular basis. Over time, market demand shortened the time between product release cycles, and subsequent advances in basic performance benchmarks became less important. In the early 1990s, recognition that this platform was not leveraging industry standard component development led IBM to transform parts of this business into a more collaborative approach. For example, the input/output (I/O) subsystem, considered to be world class 
in the industry, was able to maintain its leadership while using industry standard rather than proprietary components (fibre optic cable and connectors, optical transceivers, etc.). This led to increased interaction between development and procurement, as well as with technology suppliers outside of IBM. The operating system was another area which was opened to external developers when IBM published many of its interoperability specifications Today, a software development community exists which can port applications to both the Z/OS and Linux operating systems, university courses teach System Z skills, and a fully functional emulator for mainframe application development is available for under $\$ 100$. This has led to partnerships between hardware, firmware, and software development teams eager to optimize across traditional functional silos and exploit the full value of the server. Similarly, many companies are no longer focused exclusively on the development, manufacture and delivery of information technology, but rather on the application and integration of technology to deliver new and lasting value. The success of an innovating firm thus depends not only on its ability to meet its own innovation challenges but also on the efforts of other innovators in its environment. At the same time that partnerships have become increasingly important to IBM's business, however, the company continues to generate the largest revenue in the industry from its patent portfolio. The lucrative market for intellectual property is more often associated with the own and protect' mentality of a monolithic innovation model than a collaborative one.

Viewed in this way, the challenge for large companies becomes clearer. Large companies span both pillars of this model, and their business includes many examples of different combinations in between these extremes. Both pillars of this model have their own methodologies and business metrics for success. Tension is created when the conflicting approaches from either extreme overlap, such as when patent rights must be valued in a collaborative partnership. Along with these challenges come new opportunities; if a company is aware of these differences and can successfully balance its business by determining when to apply the proper approach and how to form creative teams, they can succeed where competitors might fail.

\section{Conceptualizing Innovation Teams}

We note that even within a monolithic culture, innovation cannot exist in a vacuum; research

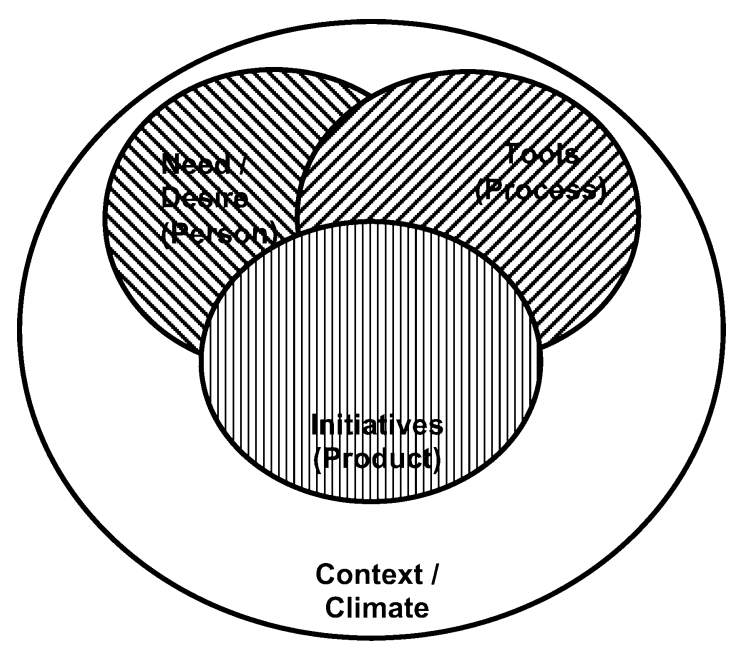

Figure 2. The Context of Innovation

scientists must work with each other to build up the necessary insights required for true innovation to occur. In a properly designed framework, this collaboration is increased and can lead to greater innovation (of course, not all partnerships are successful or well developed; today many are formed out of convenience, lacking recognition of how they apply in a broader scale). The impact of teamwork has been studied extensively (Rhodes, 1961; Johnson, 1972; Isaksen, Dorval \& Treffinger, 1994; Davila, Epstein \& Shelton, 2006); it is the nature of innovation to occur with some context, as illustrated in Figure 2. While the elements, supporting tools, processes or other elements of innovation are the focus of most efforts to enhance innovation, the overreaching context is often neglected. The context includes elements of culture, education and business climate, all of which may vary geographically or over time and are traditionally difficult to quantify. Nevertheless, without giving attention to creating a suitable context, innovation cannot flourish.

Contextual measurements are difficult to quantify, though, and it is difficult to manage what you can't effectively measure. For this reason, poor measurement has been a serious impediment to the effective management of innovation teams. Davila, Epstein and Shelton (2006) cite a recent study in which more than half of the respondents rated their performance measurement system for innovation as poor or less than adequate. This has led to lack of visibility, poor coordination, and enormous waste of money, talent, ideas and other resources. While this is clearly a serious problem with innovation in individual companies, it is even more of a problem in innovation teams, partnerships and alliances, where 
innovation spans organizational boundaries and cultures and the management complexity is truly bewildering. Spitzer (2006) discusses several keys to transforming performance measurement in teams, or in any broader organizational framework. These include a 'context' of measurement that encourages people to discover and reward innovative teamwork independent of short-term value capture, rather than use measurement to support existing preconceptions.

When measuring the impact of an innovation team, it is therefore essential to legitimize qualitative measurements. It is also important to understand how the context can be improved by building up the characteristics of collaborative innovation, not simply by increasing technical or business knowledge. Our study uncovered a good deal of research into team formation, individual achievement and group dynamics which indirectly supports these assertions. The context is sometimes referred to as the constraints applied to a problem, leading to the observation that innovation proceeds better when it is goal oriented. It has also been observed that collaboration succeeds best when it takes place between peers, with all parties feeling they have a 'winwin' situation; context is essential in establishing these roles and relationships. Thus, before we introduce a framework for categorizing different types of innovation teams, we must understand the context which will appeal to the preferences of the next generation of innovators.

\section{Innovation Teams and Generation-Y}

Each successive generation to enter the workplace bring with them a unique set of expectations and aspirations which have been shaped by their formative environment. Multigenerational workforces thus pose some unique challenges to effective team formation. Until fairly recently, the monolithic form of innovation described in Figure 1 was widely accepted as the only way to achieve significant innovation; this was reflected in the approach taken by more traditional organizations and their employees. This group consequently tended to favour conventional hierarchical learning (classroom or lecture style), and the resulting top-down communication paths were adopted in their approval-based decision making. Management chains of command were strictly observed, reinforcing the specialized role of the innovator as being confined to research or development. Indeed, such specialization was both encouraged and thought to be required, since technology was considered an unwieldy tool best left to specialists in the field. As societal norms shifted over time, the term Generation- $X$ was coined to distinguish a new workforce with different expectations, particularly regarding the role of technology and how innovation was created. With a more independent style of learning and problem solving, this generation was also empowered by increasingly easy-to-use technology. These factors contributed to more lateral communication, team building, and mentor or coach relationships in the workplace than previously. However, neither of these two generations had the benefit of being raised in an era surrounded by ubiquitous technology their entire lives; this has been a much more recent occurrence, coincident with the emergence of more collaborative innovation models.

The term Generation- $Y$ first appeared in 1993 to describe those children born between 1984 and 1994. The scope of the term has changed since then to include, in many cases, anyone born until 2001 or anyone born until the present day. Numerous alternative terms have arisen that may sometimes be regarded as sub-groups of Generation-Y. These include The Net Generation, Millennials, Second Baby Boom, My Pod generation (from the fusion of Myspace and iPod), and Generation Next. They are rapidly becoming a force for social transformation; as the next generation of innovators, it is important to understand which team-forming strategies will be best suited to Generation-Y.

There are several factors which distinguish this generation from previous ones, as noted in Table 1 (Lancaster \& Stillman, 2003). Perhaps the most significant distinction is that this is the first generation to grow up surrounded by technology and digital media. Generations are shaped by their childhood experiences and then defined by their early adulthood actions. This is the first generation to have their childhood and early adulthood influenced by trends such as the Internet, graphic interfaces and other non-keyboard access to technology, instant messaging, cell phones, digital cameras, camera-phones, sophisticated computer graphics, portable digital audio players, and robot pets. Accustomed to the strong presence of technology in their lives, immersed in it from early childhood, they are less interested in how technology works and more interested in how it may be applied to solve practical problems. Technology for them is participatory and interactive; they do not wait for anyone else to create new experiences in their technology-enabled lives; instead they create it for themselves. Broadly speaking, they see work as a means of self-actualization, rather than as a means to an end. They value 
Table 1. Innovation Characteristics of Different Generations

\section{$\begin{array}{lll}\text { Traditionalist } & \text { Gen } X & \text { Gen } Y\end{array}$}

\begin{tabular}{|c|c|c|c|}
\hline Training & The hard way & $\begin{array}{l}\text { Required to } \\
\text { keep me }\end{array}$ & Continuous and expected \\
\hline Learning style & Classroom & Independent & $\begin{array}{l}\text { Collaborative and } \\
\text { networked }\end{array}$ \\
\hline Communication style & Top down & Hub and spoke & Collaborative \\
\hline Problem-solving & Hierarchical & Independent & Collaborative \\
\hline Decision-making & Seeks approval & Team included & Team decided \\
\hline Leadership style & Command and control & Coach & Partner \\
\hline Feedback ${ }^{1}$ & No news is good news & Weekly/Daily & On demand \\
\hline Technology use & Uncomfortable & $\begin{array}{l}\text { Unable to work } \\
\text { without it }\end{array}$ & $\begin{array}{l}\text { Unfathomable if not } \\
\text { provided }\end{array}$ \\
\hline Job changing & Unwise & Necessary & Part of my daily routine \\
\hline
\end{tabular}

opportunities to be creative and exciting challenges which can make a difference to the world. They favour immediate and continual feedback, engaging early and often with their peers. The most productive innovation teambuilding strategies for this generation will be tailored to their characteristics and preferences. This makes Generation-Y a particularly fertile ground for developing collaborative innovation teams.

It is important to note that every part of the innovation value chain is strongly affected by Generation-Y, whose members have become not merely future innovators but major stakeholders in innovative value creation. Whether or not one is born as Generation-Y, each one of us is affected by their attitudes. When the workforce has an increasing proportion of Generation-Y participants, their approach will tend to influence even those from other generations of thought. The boundaries between three generations seem to be fading as technology becomes more and more user friendly. Therefore, it is becoming increasingly important to align team building to the perspective of Generation-Y, and to form new kinds of innovation teams. We will discuss four types of innovation teams, and how their approaches relate to the characteristics of Generation- $Y$ innovators.

\section{Genius Teams}

Truly radical innovation is often viewed as coming from great individual thinkers; examples such as Einstein, Da Vinci, Aristotle, and more come to mind. In reality, all of these individuals were at their most innovative when working in a community of like-minded people. We refer to these groups as 'genius teams'. Historically, genius teams are characterized by high levels of ambition and a strong positive outlook. Even if they live in difficult times and take on tough problems, they tend to believe in their ability to make things better despite the odds. Their positive outlook is created through the rules, stated goals and culture of the team. These groups are looking to make a tangible impact, with many of their efforts tied to action and driven by a desire to create change. They focus on pushing their limits in areas of high potential, and individuals in such groups identify strongly with their peers and with the group identity. Typical genius teams are small and highly selective about whom they admit into their ranks. These small inner circles nurture trust; members are highly supportive of each other and have great loyalty to the group. Despite their tendency to be mutual admiration societies, team members also seek to outdo their peers (who are seen as worthy opponents). Members actively seek recognition outside the group, often through tackling big problems and striving for the maximum possible impact.

A successful genius team is able to meet frequently in person, socialize and appreciate each other's contributions. They share and are committed to a common culture, values and rituals. The team is exclusive, tending to be small and having minimal interaction with outside organizations. They are characterized by lofty goals, positive attitude even in the face of difficulty, and a desire for recognition through celebrating goals and sharing success stories outside the team (which also helps to advance their reputation). The genius team appeals to members of Generation- $Y$ as a means of self-actualization and satisfying competitive drives, and because it provides 
immediate feedback on ideas. Because genius teams are less accepting of diversity and collaborative, team-driven decisions, however, they may not be the optimal vehicle for sustainable innovation in a Generation-Y environment.

\section{Improv Teams}

Members of genius teams are motivated by recognition from their peers and from outside the group; they tend to value 'star' performers. A different approach, which can also lead to successful innovation, values the group over the individual. The best examples of this come from improvisational (improv) teams, where all the participants feel as if they are leaders because the focus shifts among members of the group (Parker, 2003; Kail, 2004; Lublin, 2007). Improv teams are characterized by dynamic collaboration, spontaneous creativity and interaction between team members and cues taken from their surroundings. The art of improvisation requires adapting quickly to changing characters and situations based on new (or incomplete) information. Improv teams appeal to the collaborative style and social networking skills of Generation- $Y$, as well as providing rapid feedback, encouraging diversity among team members and making decisions by group consensus. However, they may not yield the highest impact results, and may also not work well with mixedgenerational teams.

Successful improvisation requires being able to accept the contributions of others, even if you do not agree with them. Improv team discussions should never backtrack; they always move forward, or branch off in a new direction not previously explored. Similar to theatrical appearances, this can be frightening to some people; it is vital to trust fellow team members to avoid destructive criticism and share the spotlight. Expressing new ideas and moving into unfamiliar directions can be intimidating or invite ridicule; this must be avoided. Improv teams take advantage of the enthusiasm of the participants; by engaging the team members and their audience, the team becomes bolder and energized. Key enablers include developing mindful presence (awareness of the audience) and willingness to either take or hand off initiative during a meeting. It is beneficial to know the motivations and interests of your team members so you can judge how your actions will affect them and develop more effective strategies for communicating with them. It is important to be aware of what other team members Oare doing to avoid wasteful (and potentially embarrassing) duplication of effort or dropping the train of thought.

\section{Virtual Teams}

Global interconnections have made the workforce more tightly integrated, making it possible for people to work from anywhere. This interaction can be encouraged through online experiences such as virtual worlds or the metaverse. This is a new generation of interactive technology, which is perhaps less effective than meeting in person but provides significantly more immersion than conference calls or email at a fraction of the cost of video conferencing or business travel. As interactive technology has become easier to use, there has been an explosive growth in the number of participants in massive online multiplayer games and virtual landscapes. IBM, Google and Linden Labs (creators of Second Life) are only a few of the companies becoming engaged in developing virtual teams for innovation and other purposes. Recent trends suggest these worlds are maturing from novelty games into potentially valuable business tools. Indeed, the user-created landscapes of Second Life serve as examples of how Generation-Y has influenced our culture. Even if those who created Second Life are not part of Generation-Y themselves, they have certainly been influenced by the innovative ideas that arose from this environment.

Virtual teams offer several advantages for Generation-Y. These environments meet their desire for social learning and deep collaboration. They also appeal to an acceptance of diversity and a meritocracy of ideas, which may actually be superior to personal interaction since it removes much of the intimidation that Generation- $Y$ associates with the more senior innovation leaders. In the metaverse your avatar can assume any appearance, keeping your real identity anonymous (if a Generation-Y team member happens to be talking with the avatar of a dog, it doesn't matter that their team member looks like a dog; it only matters what their team member has to contribute to the project). Virtual teams also appeal to Generation- $Y^{\prime}$ s reliance on technology, and may provide an opportunity to bridge the generational gap among mixed generation teams. Best practice for virtual teams includes regular meetings, supplemented by the occasional meeting in person.

As an example, a recent IBM technology conference on innovation included sessions hosted at the IBM Virtual Briefing Center in Second Life. Business conduct guidelines were developed for these teams, just as would apply in any other meeting. The team's experience in Second Life supports the assertion that virtual worlds enrich the collaborative context. Current versions continue to have significant 
barriers to entry in the form of both technical issues and time investment to learn the metaverse; these are expected to diminish over time. Despite these concerns, feedback on this experiment was very positive. Over 40 avatars attended these sessions, which enabled greater participation across global time zones. We were also able to record and videotape some of the Second Life speakers for later playback (Kristufec, 2007). Meeting content was available both in advance of the scheduled meeting time and for weeks afterwards, so that people who were not able to attend the scheduled talks could still see the material and contact speakers.

\section{FourSight Teams}

It is fundamental that a successful team will include the required skills and expertise to address the problem at hand. This same approach can be applied to the formation of innovation teams; a more structured approach to building innovation teams involves measuring team members' preferences and balancing the team accordingly. Because every individu$\mathrm{al}^{\prime}$ s personality and temperament differs, their supporting metrics are completely subjective. Instruments such as Myers Briggs Type Indicator (MBTI), Hermann Brain Dominance Instrument (HBDI) and DISC (Dominance, Influence, Steadiness, Conscientiousness) Assessment are some of the most widely known instruments for measuring personality type/temperament and cognitive thinking. The FourSight Breakthrough Thinking Profile discussed in this section differentiates itself from these instruments by building on those that measure thinking skills alone. The FourSight breakthrough thinking process, unlike psychometric instruments, is comprised of a series of discrete, repeatable steps that people regularly engage in a variety of circumstances. This means its measurement is objective. Further, the breakthrough thinking process is validated as a democratic, universal process by over 50 years of study in the field of creativity and creative problem solving (Puccio, Murdock \& Mance, 2006; Ackerbauer, 2008).

Because it is an objective measure, the breakthrough thinking process is one we can learn and intentionally replicate. If we can replicate it, we have the potential to sustain, and even scale, the results of breakthrough thinking. The more scalable (or the greater the impact of) our breakthrough thinking, the more innovative people and teams can truly be. While the creative process is universal, each step requires unique mental skills, and most individuals prefer some skills above others. Such biases show up as strong points and potential blind spots when solving problems. There are four basic preferences recognized by the breakthrough thinking model, which we will discuss in order.

1. Clarify the situation: John Dewey suggested that a problem well defined is a problem half solved. Clarifying a situation means to bring a problem, challenge or opportunity to its most granular level. If given one hour to save the world, Albert Einstein said he would spend 55 minutes understanding the problem. Clarification requires data gathering, understanding the context of a situation and asking numerous questions. Clarifying a situation can be time-intensive, because it requires a significant level of detail to ensure there are no lingering assumptions that could derail potential solutions.

2. Generate ideas: Linus Pauling said the only way to have good ideas is to have lots of ideas. Generating ideas, or fluid ideating, requires divergent thinking. Divergent thinking is about looking at the big picture, and playing with potentially abstract concepts that stretch our imagination. Because a large quantity of ideas may also breed high quality ideas, we are most effective in generating ideas when we open our minds to new thoughts, and defer judgement long enough to express and capture those ideas. Ideation, therefore, requires a more intuitive approach, whereas clarifying is most effective when employing concrete thinking.

3. Develop a solution: Developing a promising idea or series of ideas into a workable solution is about giving ideas the support required to stand on their own. Developing a solution includes comparing and analysing several noteworthy ideas in order to prioritize and strengthen one or more, then planning for their implementation. Developing a solution is about shaping raw ideas into a workable solution. Successful solution development also requires a contextual understanding of the environment, such as identifying stakeholders who will either assist or resist a solution's implementation, and taking action to amplify support and mitigate opposition.

4. Implement a plan: Implementing is nothing more than putting the plan into action. While developing a plan details what things need to happen for an idea to solve a problem, implementation is about giving structure to the idea in order for it to become a reality. Being able to successfully implement a solution requires persistence and determination. And because implemen- 
tation generally requires engaging a variety of stakeholders, implementation lends itself to re-iterating the breakthrough thinking process: Is the solution workable? Are we solving the right problem? What do we need to re-think? Who do we need on board to support this effort?

Note that qualities from each preference stand in direct contrast to those of the preference immediately preceding it. Specifically, clarification and solution development involve convergent thinking modalities, while ideation and implementation involve divergent thinking. When individuals are not aware of this distinction, conflict may arise as a result of differing approaches to problem solving. When teams are aware of their preferences, conflict can be diffused or leveraged as creative tension, producing a potentially more synergistic result. Table 2 shows the best practices for leveraging each preference when engaging teams in breakthrough thinking.

\section{Case Studies in FourSight Teams}

Although the FourSight profile does not predict performance, it does provide awareness of how teams would otherwise prefer to perform, if given the appropriate environment. FourSight has been administered to almost 300 people within IBM. Of those surveyed, approximately 75 per cent have been debriefed as to the meaning of their results. Approximately half of those have been debriefed in a formal workshop where the breakthrough thinking process was described and explored at length. In such workshops, participants are taught the breakthrough thinking process and then given their survey results. The remainder of the workshop consists of a detailed breakdown of each of the four elements of breakthrough thinking by preference name (Clarifier, Ideator, Developer and Implementer), and an introduction of critical thinking tools for leveraging that preference. We will describe one example taken from a recent IBM Academy of Technology study on innovation tools, in which the FourSight profile was administered to a self-selected innovation team; results are summarized in Figure 3.

The characteristics for each class shown in Figure 3 have been summarized previously in Table 2. Note the high preference for ideation and slight relative preference for clarification, with strong relative non-preferences for solution development and plan implementation. This suggests the team has a propensity for generating relevant ideas, yet may lack energy

Table 2. Best Practices for FourSight Team Preferences

\section{Actions}

Clarifier

Ideator

Developer

Implementer

Source: Your Thinking Profile:
(THinc Communication, 2002)

for developing and implementing strong solutions. Although the overall team was not debriefed on their preferences, some high level inferences can be drawn from the results. As a statement of preference, this team would likely generate many more ideas than there would be substantive mechanisms developed to help capture, evaluate and refine. With respect to preference, the statistical likelihood of ideas generated by this team becoming 
viable solutions, or driven to closure, is low. If the team has limited short-term goals and will then be disbanded, homogeneity may be appropriate since other teams may assume the developer and implementer roles. However, if this team were permanent, emphasis would need to be given to complementing the team's strength with a stronger solutions development focus. If this team were populated by early tenure employees, for instance, retention could be a real threat should a preponderance of ideas generated not be brought to closure.

The performance of this team bears out the profile, in that there was a great deal of challenge exploration and a rich forum for sharing thoughts and ideas during the study meetings. However, when it came to taking action and submitting the sub-team findings, there was more emphasis on ensuring ideas were captured in raw form than in a coherent summary of findings and recommendations. A series of late revisions addressed this lack of preference in idea refinement (note this is an implementation statement, proving that preferences do not necessarily predict performance). Educa-

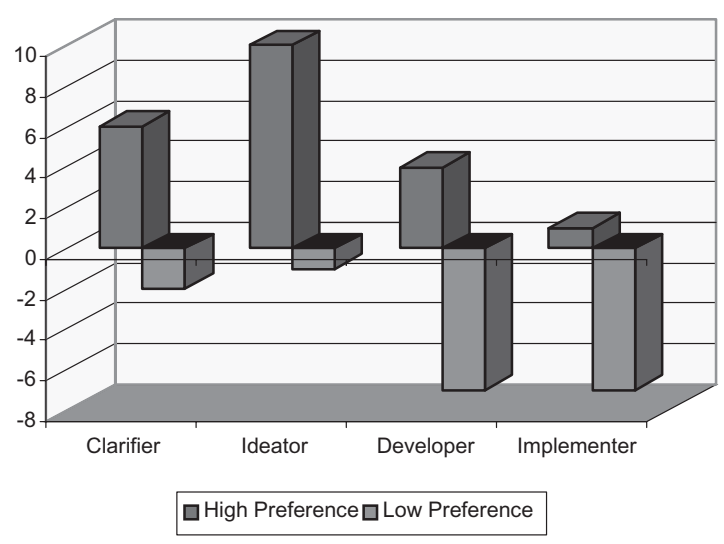

Figure 3. FourSight Team Sample Profile tion on how to prioritize and evaluate ideas, followed by driving them to closure, would have proven valuable to this team. By providing this type of preference list as part of a debriefing session, teams have immediate awareness of collective strengths, and are compelled to engage other team members so as to augment their preference gaps in the breakthrough thinking process. Teams exposed to the breakthrough thinking process have a higher likelihood of approaching problems deliberately. The more conversant teams are in the dynamics of breakthrough thinking, the more confident they are likely to be in compensating for preference gaps in the strengths of their team.

The relationship between the different types of innovation teams discussed and the characteristics of next generation innovators is summarized in Table 3. We recognize that hybrid teams incorporating the best aspects of each category might be a beneficial approach in some organizations. Furthermore, this work suggests that multi-generational teams are exposed to potential internal conflicts because of the mismatch between their preferences for different types of team participation; the analysis and treatment of such conflicts has been addressed previously in the literature (Kratzer, Leenders \& van Engelen, 2006). Additional taxonomies which extend this work to the classification of different types of innovation relationships are the subject of ongoing research (Ginsberg \& DeCusatis, 2008).

\section{Conclusions}

With the increasing emphasis on radical innovation as a differentiator, many businesses have begun to invest in building innovation teams without a clear understanding of the

Table 3. Comparison of Innovator Traits and Team Approaches

\begin{tabular}{|c|c|c|c|c|}
\hline Traits of Gen-Y Innovators & $\begin{array}{l}\text { Genius } \\
\text { Teams }\end{array}$ & $\begin{array}{l}\text { FourSight } \\
\text { Teams }\end{array}$ & $\begin{array}{l}\text { Virtual } \\
\text { Teams }\end{array}$ & $\begin{array}{c}\text { Improv } \\
\text { Teams }\end{array}$ \\
\hline Continuous learning & High & High & Medium & Medium \\
\hline Highly networked, free expression & Low & Medium & High & High \\
\hline Team decisions/no strong leader & Low & High & Medium & High \\
\hline Immediate feedback & High & Medium & High & High \\
\hline Inherent use of technology & Medium & Medium & High & Low \\
\hline Embrace diversity & Low & Medium & High & High \\
\hline Balance mixed generation team members & Medium & High & Low & Low \\
\hline Achieve self-actualization & High & High & Low & Medium \\
\hline
\end{tabular}


specific strategies which make some types of teams more likely to produce useful innovation. We have investigated different structures for teams charged with producing innovative results, including genius teams, improv teams, virtual teams and FourSight teams. Categorizing these approaches along with the preferences of Generation- $Y$ innovators, we are able to recommend strategies which are more likely to succeed because they appeal to the innovator's pre-existing motivations. Categorizing these approaches along with the preferences of Generation-Y innovators, we are able to recommend strategies which are more likely to succeed because they appeal to the innovator's pre-existing motivations. For example, we note that a Generation- $Y$ team is particularly well suited to the characteristics of a FourSight team, and relatively poorly suited to those of a genius team. We further note that preference profiling tools such as FourSight can lead to self-awareness of a team's relative strengths and weaknesses, and provide opportunities to balance the team membership to increase the prospects for long-term success.

\section{References}

Ackerbauer, M. (2008) FourSight and the Breakthrough Thinking Process. Proc. 2nd Innovation and Creativity Management Community Meeting, Buffalo, NY.

Davila, T., Epstein, M. and Shelton, R. (2006) Making Innovation Work. Wharton School Publishing, Philadelphia, PA.

Ginsberg A. and DeCusatis, C. (2008) The Innovation Ecosystem Approach to Open Innovation: Challenges and Solutions. Submitted for publication.

Ho, D. (2008) Research, Innovation, and Knowledge Management: The ICT Factor. Submitted to UNESCO (forthcoming).

Isaksen, S.G., Dorval, K.B. and Treffinger, D.J. (1994) Creative Approaches to Problem Solving. Kendall/ Hunt Publishing, Dubuque, IA.

Johnson, D.M. (1972) Systematic Introduction to the Psychology of Thinking. Harper \& Row, New York.

Kail, J. (2004) The Improv Model of Organizations [WWW document]. URL http:/ /improvatwork. com/index.php/pages/39 [accessed on 21 June 2007].

Kratzer, J., Leenders, R.Th.A.J. and van Engelen, J.M.L. (2006) Team Polarity and Creative Performance in Innovation Teams, Creativity and Innovation Management, 15, 96-104.

Kristufec, J. (2007) Improv for effective collaborative innovation, Nonconfidential session, IBM Academy of Technology conference on Innovation
Ecosystems [WWW document]. URL http:// www.ustream.tv /BorisFrampton/videos / nnndssHLIMWqVS,Saq8grg [accessed on 14 March 2008].

Lancaster, L.C. and Stillman, D. (2003) When Generations Collide: Who They Are. Why They Clash. How to Solve the Generational Puzzle at Work. Harper Business, Wheaton, IL.

Lublin, J. (2007) Improv Troupe Teaches Managers How to Give Better Presentations. The Wall Street Journal Online [WWW document]. URL http:/ / online.wsj.com/article/SB117071229131798678 [accessed on 21 June 2007].

Parker, S.G. (2003) Stand Up and Throw Away the Script. Harvard Management Communication Letter, Article Reprint No. C0302A.

Puccio, G.J., Murdock, M.C. and Mance, M. (2006) Creative Leadership: Skills that Drive Change. Sage Publications, Thousand Oaks, CA.

Rhodes, M. (1961) An Analysis of Creativity, Phi Delta Kappa, 42, 305-11.

Spitzer, D. (2006) Transforming Performance Measurement. AMACOM and The American Management Association, New York.

Dr Casimer DeCusatis is an IBM Distinguished Engineer and Technical Executive based in Poughkeepsie, NY. He is an IBM Master Inventor with over 70 patents, and co-leader of the 2007 IBM Academy of Technology study 'Innovation Ecosystems'. He is the recipient of several industry awards, including the IEEE Kiyo Tomiyasu Award, the EDN Innovator of the Year Award, the Mensa Research Foundation Copper Black Award for Creative Achievement, and the IEEE/HKN Outstanding Young Electrical Engineer award (including a citation from the President of the United States and an American flag flown in his honor over the US Capitol). He is co-author of more than 100 technical papers, book chapters, and encyclopedia articles, and editor of the Handbook of Fiber Optic Data Communication (now in its 3rd edition). Dr DeCusatis received his MS and $\mathrm{PhD}$ degrees from Rensselaer Polytechnic Institute (Troy, NY) in 1988 and 1990, respectively, and the BS degree magna cum laude in the Engineering Science Honors Program from the Pennsylvania State University (University Park, PA) in 1986. He is a member of the Order of the Engineer, a Fellow of the IEEE, Optical Society of America, and SPIE (the international optical engineering society), and a member of various other professional organizations and honor societies. 